\title{
ASSOCIATION OF CARDIORESPIRATORY FITNESS AND VASCULAR RISK ON COGNITIVE FUNCTION IN YOUNG ADULTS
}

\author{
Author \& Author
}

\begin{abstract}
The purpose of this study was to examine the interdependent associations between health indices [i.e. cardiorespiratory fitness, physical activity, body mass index (BMI)], C-reactive protein (CRP) and cognitive function in young adults. It was hypothesized that a regression analysis would confirm direct effects of the health indices and CRP on cognitive function. Health indices including cardiorespiratory fitness, physical activity level, BMI and CRP were collected to examine the relationship with cognitive function in healthy, young adults $(n=93 ; 57.0 \%$ female, aged $23.01 \pm 3.67$ years). Cognitive function was assessed using neuropsychological test battery [i.e. Kaufman Brief Intelligence Test, KBIT; Trail Making Test, (TMT); Stroop test; Psychomotor Vigilance Task, (PVT); and Delayed-Matched-to-Sample memory task, (DMS)]. Lower cognitive function of inhibitory control in the Stroop test and working memory in the DMS were observed among high CRP risk $(\mathrm{M}=5.65, \mathrm{SD} \pm 1.78)$ compared to the low CRP risk $(\mathrm{M}=0.57, \mathrm{SD} \pm 0.19)$ participants. These results suggest that CRP level in the high-risk condition causes deleterious effect on cognitive function tasks requiring inhibitory control and working memory. In addition, CRP, which was positively associated with BMI, but negatively related to cardiorespiratory fitness, had negative effects on the working memory and the inhibitory control. As predicted, higher cardiorespiratory fitness was positively associated with greater inhibitory control and working memory. Our findings demonstrated that CRP level in the high-risk condition causes deleterious effect on cognitive function among tasks demanding inhibitory control and working memory. The beneficial effect of physical exercise-induced improvements in cardiorespiratory fitness on vascular risk and cognitive functions was significant; therefore, improved cardiorespiratory fitness may reduce or suppress vascular risk with potential positive effects on cognitive health.
\end{abstract}

Keyword-cardiorespiratory fitness; C-reactive protein; cognitive function Word count: 273 


\section{INTRODUCTION}

A growing body of literature provides evidence indicating that higher levels of physical activity and cardiorespiratory fitness are associated with positive effects on cognitive and brain health (Author et al., 2014). Although the importance of physical activity across the lifespan is increasingly highlighted, inadequate physical activity, or not meeting the adult recommendations of 150 minutes of moderate to vigorous physical activity (MVPA) per week (USDHHS, 2008a), is common among young adults. This recommendation will help to maintain cardiovascular health (Pate et al., 1995), yet a recent report showed that almost two-third (74\%) of U.S. adults don't meet this guideline (USDHHS, 2008b). Insufficient physical activity is also a contributor to obesity that is related to the development of metabolic disease. Consequently, obesity has risen dramatically in U.S. adults, with more than two-thirds of individuals over the age of 20 being classified as overweight or obese in the U.S. (Ogden, Carroll, Kit, \& Flegal, 2014). Physical inactivity, lower cardiorespiratory fitness, and obesity are strong independent risk factors associated with cardiovascular disease.

C-reactive protein (CRP), known as a biomarker of systemic inflammation and an independent predictor of future cardiovascular events, is emerging as an indicator of cognitive dysfunction among older adults (Ge et al., 2013; Zhu et al., 2013). Higher levels of CRP are correlated with reduced performance on tasks involving executive function (Wersching et al., 2010). Cognitive assessments of executive function are focused on two primary measures of reaction time and accuracy of the responses (Komulainen et al., 2007; Noble et al., 2010). Cross-sectional studies (Jae et al., 2009; LaMonte et al., 2002) and a longitudinal study (Church et al., 2002) revealed an inverse relationship between higher cardiorespiratory fitness and the serum concentration of CRP, which may involve processes of cognitive function. Not only has CRP been implicated in cognitive dysfunction, but it also has been related to structural brain damage (Wersching et al., 2010).

Although the relationship between cardiorespiratory fitness and cognitive function have been observed in childhood (Hillman, Author, \& Buck, 2005), middle adulthood (Zhu et al., 2014), and late adulthood (Hayes, Forman, \& Verfaellie, 2014), the association of cardiorespiratory fitness and CRP with cognitive function in young adults is less clear. Young adult CRP-cognitive function research has been inhibited by small samples, as risk is not as prevalent in this age group (Author et al., 2013). Further, the examination of the CRP-cognition relationship has not been included in studies investigating the relationship of cognition with cardiorespiratory fitness, physical activity and BMI in young adults. Since CRP and the cardiorespiratory fitness have been reciprocally associated with cognitive function among older adults, the complex interactions among these variables should be explored among young adults given its potential for understanding how cognitive function may be preserved in later life. The CRPcognition relation is crucial for understanding cognition across the lifespan, given the increased prevalence of sedentary behaviors and the manifestation of health risk. 
Given this paucity of research and equivocal relationships, the purpose of the current investigation was to examine the interdependent associations between health indices of cardiorespiratory fitness, physical activity, body mass index (BMI), CRP and cognitive function in young adults. It was hypothesized that there would be direct effects of the health indices and CRP on cognitive function and CRP would be a key factor to explain an association between the health indices and cognitive health in young adults.

\section{INSTRUMENTS AND METHODS}

Once the study procedure was approved by The University of Texas at Austin Institutional Review Board, participants were recruited from the community via electronic forums (i.e. fliers, email, and newsletters) and word-of-mouth.

\subsection{Participants}

Healthy, English-speaking adults of either sex, of ages ranging from 18 to 30 years, and of any ethnic background were considered for the study. Participants were screened to determine if they met inclusion/exclusion criteria. The exclusion criteria for subject participation were as follows: (a) presence of cardiovascular disease or cerebrovascular disease, (b) diagnosis of psychotic disorder, (c) history of violent behavior, (d) history of neurological condition, (e) current pregnancy, and/or (f) prior institutionalization or imprisonment; however, no participant was excluded on these basis. A total of 93 participants were recruited over two years.

\subsection{Assay of High-Sensitivity CRP}

Serum high sensitivity CRP was determined using a quantitative enzyme immunoassay assay (BioCheck, Inc., USA) that includes a mouse monoclonal-CRP antibody coated onto 96-well polystyrene microplates. According to the manufacturer's assay procedure (Schultz \& Arnold, 1990), all standards, control, and samples were assayed in duplicate. Optical density of each wall was determined using a microplate reader (BioTek Inc., VT, USA) set to $450 \mathrm{~nm}$. CRP concentrations were calculated using standard curves.

\subsection{Anthropometric Measurements}

Height and weight were measured using established standards (Lohman, Roche, \& Martorell, 1988). Participant's height and weight were recorded to the nearest $0.1 \mathrm{~cm}$ and $0.1 \mathrm{~kg}$, respectively. Body Mass Index (BMI) was calculated in accordance with standard anthropometric techniques.

\subsection{Measurement of Cardiorespiratory Fitness}

For the first step, participants were appropriately positioned on the cycle ergometer (Velotron Dynafit Pro, Seattle WA). Participants then completed four incremental, two-minute stages of submaximal cycling for determination of the VO2 consumption and work rate relationship. This was followed by a bicycle ergometer test to determine maximal oxygen consumption (VO2 $\max )$. VO2 $\max$ is the greatest rate the body uses oxygen for sustained energy production, which correlates highly to endurance performance (American College of Sports Medicine (ACSM), 2013). This test required subjects to 
breathe into a spirometer for the monitoring of oxygen consumption while cycling continuously at increasing exercise intensities. The test was ended upon volitional fatigue of the subject or when cadence falls below $60 \mathrm{rpm}$. The test lasted approximately 8 to 12 minutes.

\subsection{Assessment of Physical Activity Level}

The International Physical Activity Questionnaire (IPAQ) is a 7-day physical activity recall that is based on self-reported measures of physical activity. The IPAQ instrument has acceptable measurement characteristics, particularly among this age group (Craig et al., 2003). The frequency and duration in the previous 7-days spent walking briskly and in moderate and vigorous intensity leisure-time physical activities were reported. A physical activity score was calculated as the sum of the products of total time in each of the three categories and a metabolic equivalent (MET) value assigned to each category as reported previously (Rosenberg, Bull, Marshall, Sallis, \& Bauman, 2008). This included duration $\times$ frequency per week $\times$ MET intensity: (walking $\min \times 3.0$ metabolic equivalent values) + (moderate leisure-time physical activity $\times 4.0$ metabolic equivalent values) + (vigorous leisure-time physical activity $\times 7.5$ metabolic equivalent values). A weighted estimate of total physical activity was presented as MET·min·wk1.

\subsection{Measurement of Cognitive Tasks}

Five cognitive measurements for this study included the Kaufman Brief Intelligence Test (KBIT), Stroop test, Trail Making Test (TMT), Psychomotor Vigilance Task (PVT) and Delayed-Matched-to-Sample test
(DMS), which include various domains of cognitive function such as crystallized intelligence, executive function, attention, and working memory. Except for the KBIT, each of the cognitive function tests consisted of very short (1-minute) practice trials (i.e. these data will not be recorded) in order to familiarize the participants with the task. Following this, individuals then participated in one actual trial.

2.61 KBIT. This assessment is a brief, individually administered measure of verbal and nonverbal intelligence for individuals from 4 to 90 years of age (Kaufman, 1990). The KBIT contains verbal and nonverbal subscales to estimate intelligence quotient (IQ) composite. The verbal scale is composed of two combined subtests that assess receptive vocabulary and general information as well as comprehension, reasoning, and vocabulary knowledge. The nonverbal scale uses a matrices subtest to tap the ability to complete visual analogies and to understand relationships. Participants were asked in a quiet setting to identify the object in a picture or puzzle, which served as a representation of their vocabulary. All responses required a one-word oral or signed (point with his/her finger) response within $45 \mathrm{sec}$ and took approximately 15 to 30 minutes to administer.

2.62 Stroop test. This classic test was used to examine three conditions (Word, Color, and Color-Word) by requiring the participants to respond orally to as many items as possible in 45 seconds (Stroop, 1935). The Stroop test consisted of a short (1-minute) practice trial prior to each condition (i.e. these data were not recorded) and one 
actual trial per congruent and noncongruent condition. Participants are also screened for color-blindness. For the congruent Word condition (Task A), participants were provided with a list of color words written in black ink and instructed to read orally as many words as possible. The Color condition (Task B) contained a list of "XXXX" printed in different ink colors and required the participant to say the ink color aloud. In the incongruent Color-Word condition (Task C), participants read a list of color words written in incongruent color ink relative to the printed word. The latter condition necessitates the greatest amount of interference control, as participants were required to state aloud the color of the ink and inhibit the automatic task of reading the printed word and the Stroop interference score was calculated using the following formula (Golden, 1978). Stroop Interference score $=$ Task $\mathrm{C}-[($ Task $\mathrm{A}$ $\times$ Task B $) \div($ Task $\mathrm{A}+$ Task C) $]$. The positive interference score indicates an ability to inhibit word reading, while the negative interference score represents when the word reading actively interferes with the color naming process.

2.63 TMT. This cognitive assessment was used to examine executive function through congruent and non-congruent conditions of TMT-A (i.e. low executive demand) and TMT-B (i.e. high executive demand; Smith, Servesco, \& Edwards, 2008). TMT consisted of short (1-minute) practice trial (i.e. these data were not recorded) and one actual trial. TMT-A and TMT$\mathrm{B}$ involved drawing a line connecting consecutive numbers from 1 to 25 and drawing a similar line, connecting alternating numerical and alphabetical order, respectively. For example, on
TMT-A participants draw a line from 1 to 2 to 3 , until reaching the number 25 . On TMT-B participants were asked to draw a line from 1 to $\mathrm{A}$ to 2 to $\mathrm{B}$, until reaching the final number of 25 as fast as possible. The direct score of each part was represented by the time of completion of the tasks. If an error was made during the process, it was corrected during the time sequence. The direct score of the tasks provided a difference score, which was calculated by subtracting the time (in seconds) of TMT-B from the time (in seconds) of TMT-A (Sanchez-Cubillo et al., 2009). For the data analysis, cognitive tasks were organized according to congruency in the Stroop test and executive demand in the TMT. These cognitive tasks and task conditions were organized according to congruent trials and incongruent trials in the Stroop tests, and by low executive demand and high executive demand in TMT for analysis purposes.

2.64. PVT. This cognitive task is a reaction time test for attention in which participants attend to a small fixation point at the center of a computer screen (Dinges \& Powell, 1985). At random intervals, a bright millisecond timer appears in the center of the rectangle. Participants were given short (1-minute) practice trials of the PVT to familiarize them with the task. Participants were instructed to respond via button press as rapidly as possible upon detection of the counter stimulus (i.e. participant response stops the counter from updating). The final counter value corresponded to the participant's reaction time. The success or failure trial is displayed on-screen for 1-second, thus providing feedback for that particular trial. Participants were given 30-seconds to make a response before the computer aborts a trial. Information about each trial's reaction time, and success or 
failure, was stored by the computer for later analysis. The block of PVT trials was approximately five minutes long. Because between trial intervals are pseudo-randomly chosen without replacement from between two and 10seconds or an average of six seconds. The test was organized into blocks of five minutes and consisted of approximately 45 trials. After five minutes, the block of trials terminated, regardless of how many trials had elapsed (the post-treatment block of PVT trials was identical to the first).

2.65. DMS. The task is measured in both memory retrieval latency and correct match-to-sample trials (response accuracy; Chudasama \& Yogita, 2010). Participants were also given short (1minute) practice trials of the DMS to familiarize them with the task. Participants viewed a $5 \times 5$ grid of brightly colored yellow and red squares with a unique pattern. Then, with the press of a key, the stimulus disappeared and the screen was blank throughout a delay period (6 seconds). Two stimuli then were presented on the screen (a "match" and "non-match"). Participants were asked to indicate which stimulus was the correct "match" with a key press and to respond as quickly and as accurately as possible. Correct match-tosample trials and memory retrieval latency were measured by the computer and stored for later analysis. The average between trial intervals in the DMS was 7.5 seconds, so depending on how long the subject examines each post-delay stimulus to choose the "match", which took approximately 5-6 minutes. There were 30 trials per block. The PVT and DMS were implemented with a program called the Psychology Experiment Building Language (PEBL), an opensource programming language that can be run on any Windows computer (Mueller \& Piper, 2014). One desktop computer in a closed office was designated as the testing apparatus. These data gathered by the PEBL program as a .txt file output, which includes each trial's between trial interval in seconds, reaction time and study time in milliseconds, and a code number indicating whether the trial was a success (response in less than 30 seconds), a lapse (no response in 30 seconds), or a false alarm (responded with a button press prior to the onset of the cue). Participants were identified by a code number (a randomly assigned subject number), which was typed into the program prior to the start of each block of trials.

\subsection{Study Procedure}

The study consisted of two testing days of approximately 90-mins each, with a maximum of seven days between sessions (see Figure 1). On a day one of testing, the participants reported to the laboratory after having refrained from strenuous exercise and alcoholic beverages for 24 hours and from caffeine and food for 12 hours. All participants signed the informed consent for participation in this study and immediately completed a health research screening survey. After blood pressure was measured, an approximate $10 \mathrm{ml}$ blood sample was obtained from venipuncture of the non-dominant arm. Once a whole blood sample collected, blood sample was immediately transferred into the red-topped tubes and were centrifuged for 10 minutes at $3000 \mathrm{rpm}$ at $4^{\circ} \mathrm{C}$ in a Sorvall RC-6 centrifuge (Thermo Fisher Scientific Inc, Waltham, Mass, USA). After centrifugation, $0.6 \mathrm{~mL}$ of serum were transferred to new three $12 \times 75-\mathrm{mm}$ test tubes and immediately stored at $-80^{\circ} \mathrm{C}$ for analysis of CRP. Once the 
blood samples were being analyzed the blood were discarded following appropriate bio-safety procedures. Following the blood sample draws, participant's height and weight were assessed and then participants performed baseline cognitive testing on KBIT, Stroop, and TMT, in this order.
Following the completion of cognitive testing, participants performed a maximal graded exercise test to volitional fatigue on a cycle ergometer (Velotron Dynafit Pro, Seattle WA) to measure participants' VO2 max, which is considered to be the criterion measure for cardiorespiratory fitness (American College of Sports Medicine, 2000).

\begin{tabular}{l|l|l|l|l|l|l}
\hline \multicolumn{5}{c|}{ Day 1 } & \multicolumn{1}{c}{ Day 2 } \\
\hline $\begin{array}{l}\text { Consent } \\
\text { form } \\
\bullet \begin{array}{l}\text { Health } \\
\text { Screening }\end{array}\end{array}$ & $\rightarrow$ & $\begin{array}{l}\bullet \text { Blood } \\
\text { pressure } \\
\begin{array}{l}\text { Height \& } \\
\text { weight } \\
\text { Blood } \\
\text { sample }\end{array}\end{array}$ & $\rightarrow$ & $\begin{array}{l}\bullet \text { KBIT } \\
\bullet \text { Stroop } \\
\bullet \text { TMT }\end{array}$ & $\bullet \mathrm{VO}_{2}$ max & $\bullet$ PVT \\
$\bullet$ DMS \\
\hline
\end{tabular}

Figure 1. Experimental Protocol Sequence

Abbreviation: Kaufman Brief Intelligence Test, KBIT; Trail making test, TMT; PVT, Psychomotor vigilance task; DMS, Delayed-match-to- sample task.

On day two of the testing, participants also reported to the laboratory after having refrained from strenuous exercise and alcoholic beverages for 24 hours and from caffeine and food for 12 hours. Participants completed baseline cognitive testing including the PVT and DMS task, in this order.

\subsection{Data Analysis}

Statistical analyses were performed using SPSS Statistical Packages v21 (SPSS Inc, Chicago, USA). First, it was determined whether each dependent variable was normally distributed, by assessing its skewness and kurtosis. Descriptive statistics were used to represent characteristics of the participants and to confirm data entry. Mean differences in dichotomous variables (i.e. gender) and other sample characteristics (i.e. ethnicity, BMI category) for CRP (mg/L) were compared using independent t-tests. Correlations were conducted and significantly associated variables were used as covariates in the subsequent analyses. A one-way analysis of variance was employed to compare mean differences of cognitive variables on three CRP risk levels such as low risk $(<1.0 \mathrm{mg} / \mathrm{L} ; \mathrm{n}=39)$, intermediate risk (1.0 to $3.0 \mathrm{mg} / \mathrm{L} ; \mathrm{n}=20)$, and high risk (>3.0 mg/L; $\mathrm{n}=21)$. Post hoc analyses using the LSD were performed to evaluate the mean difference. A multiple linear regression analysis was used to examine the associations of health indices upon CRP and cognitive variables and develop a model for predicting: (a) CRP level from health indices, (b) each cognitive variable from CRP level, and (c) each cognitive variable from health indices without (model 1) and with (model 2) adjustment for age, gender, and race.

\section{RESULTS}

Of the 98 volunteers who participated 
in the study, five were excluded because of incomplete data, leaving a total of 93 (95\%) available for subsequent analyses. Sample characteristics are presented in Table 1. Seventy-five percent of participants were of normal weight $(\mathrm{BMI}<25)$ and $25 \%$ of participants were overweight or obese (BMI > 25). In gender comparison, male participants $(n=40)$ had significantly higher BMI $(P=0.002), \quad$ waist circumstance $(P=0.001)$, systemic blood pressure $(P<0.001)$ and VO2 max $(P<0.001)$, compared to female participants $(n=53)$.

Table 1.

Participants Characteristics

\begin{tabular}{|c|c|c|c|c|c|c|}
\hline \multirow[t]{2}{*}{ Variable } & \multicolumn{2}{|c|}{ Total $(n=93)$} & \multicolumn{2}{|c|}{ Male $(n=40)$} & \multicolumn{2}{|c|}{ Female $(n=53)$} \\
\hline & Mean & SD & Mean & SD & Mean & SD \\
\hline Age, yrs. & 23.01 & 3.68 & 23.73 & 3.64 & 22.47 & 3.64 \\
\hline Height, cm & 169.54 & 9.23 & $177.26^{* *}$ & 6.20 & 163.86 & 6.58 \\
\hline Weight, kg & 65.17 & 13.61 & $74.62 * *$ & 12.89 & 58.04 & 9.08 \\
\hline BMI & 22.51 & 3.33 & $23.71 * *$ & 3.20 & 21.62 & 3.17 \\
\hline $25<$ BMI, n (\%) & 75 & & 72.5 & & 88.6 & \\
\hline 25>BMI, n (\%) & 25 & & 27.5 & & 11.3 & \\
\hline Waist circumstance, cm & 77.90 & 9.14 & $82.30 * *$ & 9.20 & 74.92 & 7.91 \\
\hline $\mathrm{SBP}, \mathrm{mmHg}$ & 109.11 & 12.18 & $117.22^{* *}$ & 11.71 & 102.87 & 8.28 \\
\hline $\mathrm{DBP}, \mathrm{mmHg}$ & 73.05 & 8.35 & 74.38 & 8.81 & 72.04 & 7.91 \\
\hline $\mathrm{MET} \cdot \min \cdot \mathrm{wk}-1$ & 697.41 & 415.26 & 772.23 & 431.76 & 646.22 & 401.28 \\
\hline $\mathrm{VO}_{2} \max \mathrm{ml} . \mathrm{kg} / \mathrm{min}$ & 37.78 & 9.63 & $43.93^{* *}$ & 9.36 & 33.23 & 6.98 \\
\hline HRmax, beats/min & 181.06 & 13.54 & 181.81 & 13.99 & 180.50 & 13.31 \\
\hline $\mathrm{RER}, \mathrm{CO}_{2} / \mathrm{O}_{2}$ & 1.11 & 0.09 & 1.11 & 0.09 & 1.11 & 0.10 \\
\hline $\mathrm{CRP}, \mathrm{mg} / \mathrm{L}$ & 1.84 & 1.91 & 1.87 & 2.21 & 1.80 & 1.63 \\
\hline \multicolumn{7}{|l|}{ Ethnicity (\%) } \\
\hline Caucasian & $37 \%$ & & $19 \%$ & & $18 \%$ & \\
\hline Asian & $35 \%$ & & $4 \%$ & & 6 & \\
\hline African & $10 \%$ & & $13 \%$ & & $22 \%$ & \\
\hline Hispanic & $8 \%$ & & $2 \%$ & & $6 \%$ & \\
\hline Other & $3 \%$ & & $2 \%$ & & $1 \%$ & \\
\hline
\end{tabular}

Abbreviations: BMI, body mass index, SBP, systolic blood pressure, DBP, diastolic blood pressure, VO2max, maximal oxygen consumption; HR, heart rate; MET, metabolic equivalent; RER, respiratory exchange ratio; CRP, C-reactive protein.

Asterisks in the Male column indicate sex differences.

$* \mathrm{P}<0.05 ; * * \mathrm{P}<0.01$

\subsection{Correlation Between Health Indices and Cognitive Variables}

Table 2 displays the correlations of all variables. CRP was significantly correlated with BMI $(r=0.25, P=$ 0.009), waist circumstance $(r=0.43, P$ $=0.003)$, suggesting a positive relationship of CRP with BMI and waist circumstance. Among the cognitive variables, CRP is significantly related to scores in Stroop color word $(r=-0.27, P$ $=0.02)$ and Stroop interference $(r=-$ $0.29, P=0.012$ ) for executive function and memory retrieval latency in DMS test $(r=0.37, P=0.007)$ for working memory, thus indicating higher CRP is negatively related to inhibitory control 
and working memory. There is no significant relationship between CRP and other cognitive variables such as KBIT composite representing crystallized intelligence $(r=0.22, P=$ $0.089)$, PVT for attention $(r=0.18, P=$ 0.237), and TMT B-A for executive control $(r=-0.13, P=0.237)$. In contrast, there was significant correlation between $\mathrm{VO} 2 \mathrm{max}$ and memory retrieval latency $(r=-0.32, P=$ 0.012) and correct match-to-sample trials $(r=0.29, P=0.023)$ in DMS test for working memory and reaction time and $(r=-0.26, P=0.047)$ in PVT for sustained attention suggesting a greater working memory control relative to high cardiorespiratory fitness. In addition, physical activity level was significantly related to memory retrieval latency ( $r=$ -0.30, $P=0.021)$ in DMS test and reaction time $(r=-0.28, P=0.026)$ in PVT. Further, physical activity level was also significantly related to inhibitory control as indicated by a positive correlation with Stroop Color Word $(r=$ $0.30, P=0.015)$ and interference $(r=$ $0.28, P=0.028)$. Significant correlation between VO2max and physical activity level was observed $(r=0.30, P=0.016)$, suggesting a more intense physical activity participation might improve or maintain cardiorespiratory fitness level.

Table 2. Correlations of Variables

\begin{tabular}{|c|c|c|c|c|c|c|c|}
\hline Variables & Mean (SD) & Age & BMI & WC & $\mathrm{VO}_{2} \max$ & MET & CRP \\
\hline Age, year & $23.68(3.68)$ & - & & & & & \\
\hline BMI & 22.51 (3.33) & 0.07 & - & & & & \\
\hline WC & $77.9(9.14)$ & -0.07 & $0.76 * *$ & - & & & \\
\hline $\mathrm{VO}_{2} \max$ & $37.78(9.63)$ & -0.04 & 0.03 & 0.06 & - & & \\
\hline $\mathrm{MET} \cdot \min \cdot \mathrm{wk}-1$ & $\begin{array}{l}697.41 \\
(415.25)\end{array}$ & -0.13 & 0.06 & 0.07 & $0.30 *$ & - & \\
\hline $\begin{array}{l}\text { CRP } \\
\text { KBIT }\end{array}$ & $1.83(1.91)$ & 0.03 & $0.25^{*}$ & $0.43^{* *}$ & -0.12 & -0.23 & - \\
\hline KBIT Vocabulary & $\begin{array}{l}102.06 \\
(12.24)\end{array}$ & -0.18 & 0.02 & -0.02 & 0.14 & 0.05 & $0.25^{*}$ \\
\hline KBIT Mat & 106.39 (6.99) & $0.28^{* *}$ & 0.02 & -0.07 & 0.18 & 0.04 & 0.01 \\
\hline KBIT Composition & $104.59(8.17)$ & -0.03 & 0.03 & -0.05 & 0.20 & 0.06 & 0.22 \\
\hline \multicolumn{8}{|l|}{ Stroop test } \\
\hline Stroop Word (score) & $\begin{array}{l}107.32 \\
(14.90)\end{array}$ & -0.16 & 0.17 & 0.07 & 0.12 & -0.04 & -0.08 \\
\hline Stroop Color (score) & 78.65 (12.74) & $-0.35^{* *}$ & 0.13 & -0.01 & 0.18 & 0.22 & -0.08 \\
\hline Stroop Color Word (score) & $53.01(10.83$ & -0.15 & 0.03 & 0.00 & 0.19 & $0.30 *$ & $-0.27 *$ \\
\hline Stroop Interference (score) & $7.81(8.73)$ & 0.06 & -0.08 & -0.02 & 0.10 & $0.28^{*}$ & $-0.29 *$ \\
\hline \multicolumn{8}{|l|}{ TMT } \\
\hline TMT A (sec) & $15.79(5.03)$ & -0.03 & 0.12 & 0.23 & -0.13 & -0.11 & 0.20 \\
\hline TMT B (sec) & $38.26(11.46)$ & 0.05 & -0.13 & -0.13 & -0.09 & -0.09 & -0.03 \\
\hline TMT B-A (sec) & $22.10(10.07)$ & 0.10 & -0.18 & $-.310 *$ & -0.02 & -0.01 & -0.13 \\
\hline \multicolumn{8}{|l|}{ PVT } \\
\hline Reaction time (msec) & $\begin{array}{l}358.38 \\
(27.23)\end{array}$ & -0.10 & -0.00 & -0.12 & $-0.26^{*}$ & $-0.29 *$ & 0.18 \\
\hline \multicolumn{8}{|l|}{ DMS } \\
\hline Correct trial (score) & $27.06(1.96)$ & 0.19 & -0.12 & 0.06 & $0.29 *$ & 0.11 & $-0.32 *$ \\
\hline Reaction time (msec) & $\begin{array}{l}1931.70 \\
(590.20)\end{array}$ & -0.13 & 0.01 & 0.15 & $-0.32 *$ & $-0.30 *$ & $0.37 * *$ \\
\hline
\end{tabular}

Abbreviations: KBIT, Kaufman Brief Intelligence Test; BMI, body mass index; WC, waist circumstance; VO2max, maximal oxygen consumption; MET, metabolic equivalent; CRP, Creactive protein; TMT, Trail Making Test; PVT, Psychomotor vigilance task; DMS, Delayed Copyright (C) 2015, Knowledge Enterprises Incorporated. All rights reserved. 


\subsection{C-Reactive Protein Risk Level and Cognitive Function}

Figure 2 displays the mean difference of Stroop interference (score) in Stroop test and memory

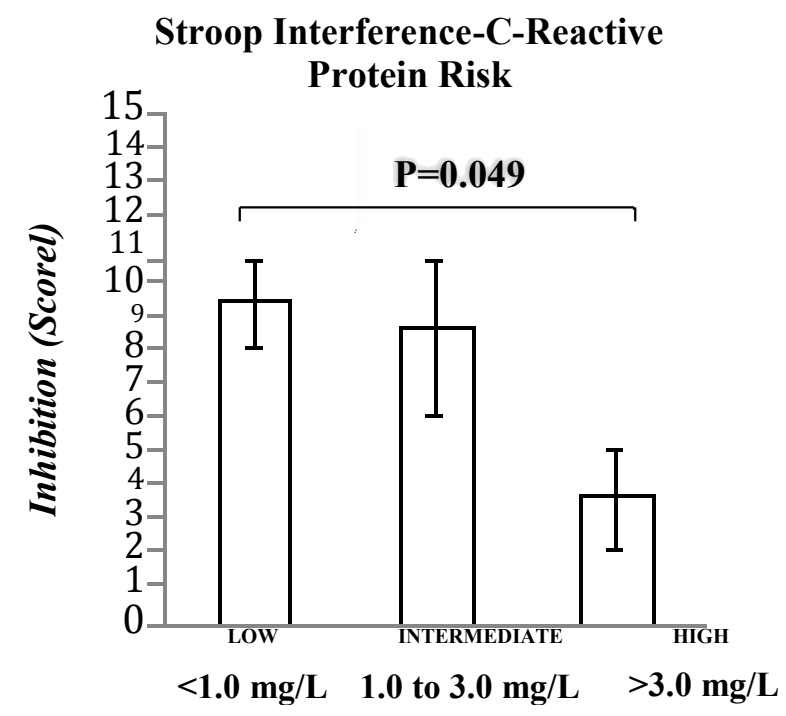

retrieval latency (msec) in DMS on three CRP risk levels such as low risk $(<1.0 \mathrm{mg} / \mathrm{L} ; \mathrm{n}=39)$, intermediate risk (1.0 to $3.0 \mathrm{mg} / \mathrm{L} ; \mathrm{n}=20)$, and high risk (>3.0 mg/L; $\mathrm{n}=21)$.

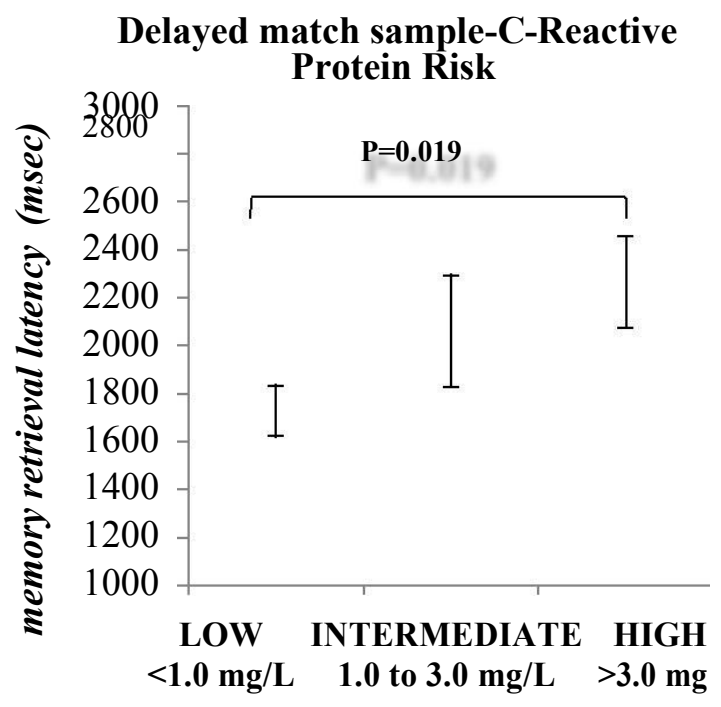

Figure 2. C-Reactive Protein Risk-Stroop Interference and Memory Retrieval Latency

The mean difference of Stroop interference (score) in Stroop test indicating the inhibitory control for three CRP risks are shown in (left). The mean difference of Memory Retrieval Latency (msec) in Delayed match sample test indicating the working memory for three CRP risks are shown in (right). Data are presented as Mean (standard errors).

One-way ANOVA analysis revealed the effect of CRP risk was significant on Stroop interference in Stroop test $(F 2,77=2.93, P=0.049)$ and memory retrieval latency in DMS $(F 2,77=$ 3.32, $P=0.05$; see Table 3). Post hoc analyses using the LSD post hoc criterion for significance indicated that the average scores in the Stroop interference was significantly lower in the high CRP risk $(\mathrm{M}=3.61, \mathrm{SD} \pm$ 6.95) than in the low CRP risk $(\mathrm{M}=$ $9.41, \mathrm{SD} \pm 8.21)$. In addition, average time in memory retrieval latency (msec) in DMS was significantly slower in the high CRP risk $(\mathrm{M}=$ 2265.96, SD \pm 610.89$)$ than in the low $\mathrm{CRP}$ risk $(\mathrm{M}=1727.31, \mathrm{SD} \pm 601.42)$.
These results suggest that CRP level in the high-risk condition causes deleterious effect on cognitive function especially such as inhibitory control and working memory. 
Table 3. Mean Difference of Variables on Levels of C-Reactive Protein Risk

\begin{tabular}{|c|c|c|c|c|c|c|c|c|}
\hline \multirow[t]{2}{*}{ Variables } & \multicolumn{2}{|c|}{$\begin{array}{c}\text { Low Risk } \\
<1.0 \mathrm{mg} / \mathrm{L} \\
\mathrm{N}=39\end{array}$} & \multicolumn{2}{|c|}{$\begin{array}{c}\text { Intermediate Risk } \\
1.0 \text { to } 3.0 \mathrm{mg} / \mathrm{L} \\
\mathrm{N}=20\end{array}$} & \multicolumn{2}{|c|}{$\begin{array}{c}\text { High Risk } \\
>3.0 \mathrm{mg} / \mathrm{L} \\
\mathrm{N}=21 \\
\end{array}$} & \multirow[t]{2}{*}{$F$} & \multirow[t]{2}{*}{$P$} \\
\hline & Mean & SD & Mean & SD & Mean & SD & & \\
\hline C-reactive protein, $\mathrm{mg} / \mathrm{L}$ & 0.57 & 0.19 & 1.77 & 0.58 & 4.65 & 1.78 & 120.02 & 0.00 \\
\hline Body Mass Index, kg/m2 & 22.06 & 2.23 & $23.97^{*}$ & 3.30 & $24.31 * *$ & 4.43 & 4.12 & 0.02 \\
\hline Waist Circumstance, $\mathrm{cm}$ & 75.58 & 6.09 & $85.74 * *$ & 7.01 & $84.30 * *$ & 13.21 & 7.71 & 0.00 \\
\hline VO2 $\max , \mathrm{ml} / \mathrm{kg} / \mathrm{min}$ & 40.00 & 9.83 & 37.61 & 10.73 & 34.76 & 11.16 & 1.50 & 0.23 \\
\hline PA level, MET·min·wk-1 & 754.30 & 434.87 & 567.25 & 416.80 & 554.00 & 315.55 & 1.29 & 0.29 \\
\hline \multicolumn{9}{|l|}{ Cognitive variable } \\
\hline \multicolumn{9}{|l|}{ Crystallized intelligence } \\
\hline KBIT Vocabulary & 99.36 & 15.35 & 102.00 & 10.17 & 106.94 & 8.17 & 2.15 & 0.13 \\
\hline KBIT Mat & 106.56 & 7.98 & 107.59 & 6.97 & 105.67 & 4.90 & 0.32 & 0.73 \\
\hline KBIT Composition & 103.13 & 10.15 & 105.24 & 6.49 & 106.94 & 5.84 & 1.31 & 0.28 \\
\hline Stroop test & & & & & 0.82 & & & \\
\hline Stroop Word (score) & 106.79 & 17.29 & 110.88 & 15.28 & 105.78 & 15.58 & 0.49 & 0.61 \\
\hline Stroop Color (score) & 78.00 & 15.99 & 78.88 & 9.76 & 77.39 & 9.51 & 0.06 & 0.95 \\
\hline Stroop Color Word (score) & 54.13 & 11.59 & 54.65 & 12.17 & 48.22 & 8.12 & 2.08 & 0.13 \\
\hline Stroop Interference (score) & 9.41 & 8.21 & 8.76 & 10.78 & $3.61^{*}$ & 6.95 & 2.93 & 0.05 \\
\hline \multicolumn{9}{|l|}{ Trail taking test } \\
\hline Trail Making A (sec) & 14.76 & 4.73 & 16.54 & 4.75 & 16.30 & 5.68 & 1.03 & 0.36 \\
\hline Trail Making B (sec) & 36.27 & 10.94 & 42.09 & 13.58 & $34.26^{*}$ & 7.06 & 2.53 & 0.09 \\
\hline Trail Making B-A (sec) & 21.51 & 9.15 & 25.55 & 13.36 & $17.96^{*}$ & 8.14 & 2.50 & 0.09 \\
\hline \multicolumn{9}{|l|}{ Psychomotor vigilance task } \\
\hline Reaction time (msec) & 343.97 & 67.23 & 352.75 & 26.29 & 364.61 & 38.06 & 0.50 & 0.61 \\
\hline \multicolumn{9}{|l|}{ Delayed match sample test } \\
\hline Correct trial (score) & 26.44 & 5.24 & 27.38 & 1.60 & $26.30^{*}$ & 2.31 & 0.17 & 0.85 \\
\hline Reaction time (msec) & 1727.31 & 601.42 & 2060.42 & 660.61 & 2265.96 & 610.89 & 3.32 & 0.05 \\
\hline
\end{tabular}

Abbreviations: BMI, body mass index; VO2max, maximal oxygen consumption; MET, metabolic equivalent; CRP, C-reactive protein.

Data are presented as mean \pm SD; asterisks indicate differences in levels of C-reactive protein risk

$* \mathrm{P}<0.05 ; * * \mathrm{P}<0.01$

\subsection{Predictor Variables on Cognitive}

\section{Function}

Multiple linear regression analysis was used to develop a model for predicting:

(a) CRP level from health indices (i.e. BMI, VO2max, physical activity level), (b) cognitive variables (i.e. inhibition, attention, working memory) from CRP level, and (c) cognitive variables from health indices without (model 1) and with (model 2) adjustment for age, gender, and race.

The multiple regression analysis was used to test if health indices significantly predicted CRP. Two predictors such as BMI and VO2max explained $39.1 \%$ of the variance $\left(R^{2}=0.38, F 3,76=9.622, P\right.$ $=0.001$ : Model 1) and $43.0 \%$ of the variance $\left(R^{2}=.43, F 3,76=5.286, P=\right.$ 0.001: Model 2) after adjustment for age, 
gender, and race. CRP was significantly predicted from increased BMI $(\beta=0.46$, $P=0.001)$, while significantly related to decreased cardiorespiratory fitness $(\beta=-$ $0.57, \mathrm{P}=0.003)$. Although physical activity level was not significantly related to CRP, increased physical activity level was significantly associated with increased cardiovascular fitness $(\beta=0.19, P=0.048)$ in the model $\left(R^{2}=0.49, F 4,82=15.15, P<\right.$ 0.001: Model 2).

The multiple regression analysis was used to test if CRP significantly predicted each cognitive variable. After adjustment for age, gender, and race, $\mathrm{CRP}$ as a predictor explained $17.8 \%$ of the variance $\left(R^{2}=0.178, F 4,74=5.286\right.$ $P=0.05$ : Model 2) and significantly predicted memory retrieval latency in DMS $(\beta=0.378, P=0.007)$. In addition, CRP significantly predicted score in Stroop interference $(\beta=-0.27, \mathrm{P}=0.02)$ although it did not have significant effect in the model $\left(R^{2}=.1, F 4,74=2.294\right.$ $P=0.06$ : Model 2), but it showed a significant trend. However, CRP did not predict the KBIT $(\beta=0.18, P=0.107)$ in the Model $2\left(R^{2}=.144, F 4,74=5.286\right.$ $P=0.028)$ and completion time in TMT $\mathrm{B}-\mathrm{A}(\beta=-0.12, \mathrm{P}=0.286)$ in the Model $2\left(R^{2}=0.02, F 4.74=0.353 P=0.841\right.$ : Model 2), and reaction time in PVT $(\beta=$ $0.166, \mathrm{P}=0.269)$ in the Model $2\left(R^{2}=\right.$ $.031, F 4.74=0.356 P=0.839$ : Model 2). These results indicated the increased CRP had the significant negative direct effects on memory retrieval latency (msec) in DMS for working memory and scores in Stroop interference for inhibitory control while there was no significant effect on reaction time in PVT for attention, completion time in TMT for executive function, and scores in KBIT for crystalized intelligence.

The multiple regression analysis was used to test if health indices significantly predicted each cognitive variable. Two predictors of $\mathrm{VO} 2 \mathrm{max}$ and physical activity level explained $12.7 \%$ of the variance $\left(R^{2}=0.127, \mathrm{~F} 3,83=2.757, P=\right.$
0.051: Model 1) and $18.6 \%$ of the variance $\left(R^{2}=0.186, F 6,80=2.062, P=\right.$ 0.06: Model 2) after adjustment for age, gender, and race. Memory retrieval latency in DMS was significantly predicted from increased VO2max $(\beta=$ $0.37, P=0.04)$ and weakly predicted from increased physical activity level $(\beta$ $=-0.24, P=0.07)$, but it was not significantly predicted from increased BMI $(\beta=0.06, P=0.66)$. In addition, score in Stroop interference was significantly predicted from increased physical activity level $(\beta=0.26, P=$ $0.05)$ although it did not have significant effect in the model $\left(R^{2}=0.168, F 6,80=\right.$ 1.848, $P=0.107$ : Model 2). No health variable predicted crystalized intelligence in KBIT $\left(R^{2}=0.222, F 6.80\right.$ $=0.494, P=0.810)$, score in Stroop interference $\left(R^{2}=0.168, F 6,80=1.848\right.$, $P=0.107)$ completion time in TMT B-A $\left(R^{2}=0.201, \quad F 6,80=2.26, P=0.06:\right.$ Model 2), and reaction time in PVT $\left(R^{2}\right.$ $=0.067, F 6,80=0.647 P=0.692$ : Model $2)$. These results indicated the increased VO2max and physical activity level had significant, negative, direct effects on memory retrieval latency $(\mathrm{msec})$ in DMS for working memory, suggesting a faster control process of working memory among fit and healthy participants. Additionally, the increased physical activity level was related to greater inhibitory control. Using the predictors that emerged from the regression analysis, cardiorespiratory fitness level (VO2max) had significant, negative, direct effects on CRP $(\beta=-$ $0.57, \mathrm{P}=0.003)$ associated with $\mathrm{BMI}(\beta$ $=0.46, P=0.001)$. Although there was no direct relationship between physical activity level and CRP, a positive relationship between cardiorespiratory fitness level and physical activity level $(\beta=0.19, P=0.048)$. Positive and significant direct effects were found for those who maintained cardiorespiratory fitness level $(\beta=-0.37, P=0.04)$ on working memory. A significant negative direct effect was observed for CRP on 
working memory $(\beta=0.378, P=0.007)$. However, no significant negative direct effect was found for BMI on working memory $(\beta=0.06, P=0.66)$ in the model $\left(R^{2}=.186, F 6.80=2.062, P=\right.$ 0.06: Model 2). When CRP was considered as a potential mediator of the relationship between BMI and working memory, the indirect effect of BMI on working memory might be dependent on CRP level.

\section{DISCUSSION}

The purpose of this study was to examine the relations of various health indices, physical activity, physical fitness, and cognitive performance among, seemingly healthy young adults in the second decade of life. This study is timely and warranted because the relationship between such variables have been largely unexamined since this age group is considered to be at their cognitive peak and have few differences in reaction time and accuracy (Whiteman et al., 2014). Given the paucity of research, the findings from this study help to establish a distinct and needed area of study in this portion of the lifespan, when health risk potentially manifests asymptomatic disease.

\subsection{CRP and Health Indices}

Consistent with previous research, this study found that the potential beneficial effects of regular physical activity participation and a healthy body weight on the vascular and cognitive health of young adults have been previously highlighted (Zhu et al., 2014; Zhu et al., 2013). Evidence suggests that physical inactivity, pooper cardiorespiratory fitness, or obesity increases CRP levels and impairs cognitive dysfunction. This study is the first known to examine cardiorespiratory fitness, health risk and executive function among, self-reporting healthy, young adults. Furthermore, our study contributes to the existing literature by assessing CRP as a potential mediator of the relationships between cardiorespiratory fitness and cognitive function. The results of this present study confirmed the relationship of cardiorespiratory fitness, physical activity, and BMI and CRP (Ford, 2002; Kasapis \& Thompson, 2005) with cognitive function in that was previously found among older adults.

Specifically, the findings from adjusted regression analysis showed that cardiorespiratory fitness and BMI were the strongest predictors of altered CRP level. Although our participants are healthy young and mostly within a normal range of BMI, BMI was significantly a predictor of the increased CRP level. This finding is consistent with a previous a study demonstrating that CRP predicts future risk for cardiovascular disease in apparently healthy individuals (Jialal, Devaraj, \& Venugopal, 2004). BMI commonly is a strong predictor of CRP levels relative to other lipid profiles such as triglyceride and cholesterol, suggesting that obesity is the major factor associated with elevated CRP in individuals by increasing adipose tissue that produces proinflammatory cytokines such as tumor necrosis factoralpha and interleukin-6 (Hotamisligil, Arner, Caro, Atkinson, \& Spiegelman, 1995). Particularly, interleukin-6 is the major stimulator of the production of CRP in the liver (Heinrich, Castell, \& Andus, 1990). In contrast, a previous study demonstrated an inverse relationship of physical activity and cardiorespiratory fitness and the serum concentration of inflammatory markers with CRP (Kasapis \& Thompson, 2005). The inverse association between physical activity and CRP levels was 
dependent on greater amount of leisuretime physical activity (Ford, 2002) and higher levels of physical activity (Albert, Glynn, \& Ridker, 2004). Yet, in the present study, physical activity level based on a self-reported data was not significantly related to a deceased CRP level possibly due to the self-reported data and reporting biases, which undermine the accurate classification of physical activity. In the present, however, the physical activity level was positively associated with cardiorespiratory fitness level as the most accurate measure of eliminating self-reporting bias. The data from adjusted regression analysis demonstrated that cardiorespiratory fitness level has the strongest negative effect on CRP level. This finding consistently matches with previous studies reporting an inverse association between cardiorespiratory fitness level and CRP level (Ford, 2002; Kasapis \& Thompson, 2005). The beneficial effects of chronic physical activity and cardiorespiratory fitness on reducing or suppressing systemic CRP levels by potential mechanisms, including a decrease in proinflammatory cytokine production by adipose tissue, skeletal muscles, endothelial and blood mononuclear cells, a improved endothelial function and an antioxidant effect (Kasapis \& Thompson, 2005). Although the data from the adjusted regression analysis highlight the potential pathways of health indices on CRP and cognitive function, our findings do not establish a causal relationship. Therefore, having higher cardiorespiratory fitness level and lower BMI may result in lowered CRP level, thus mediating cognitive function ( $\mathrm{Ge}$ et al., 2013; Zhu et al., 2013).

\subsection{CRP and Cognitive Function}

Previous studies have shown that cardiorespiratory fitness was independently associated with CRP and cognitive function (Komulainen et al., 2007; Noble et al., 2010). Parallel with this association is evidence linking CRP levels with cognitive function. Our adjusted regression analysis demonstrated significant negative relationships between CRP level and cognitive function particularly such as inhibitory control and working memory. These relationships were reinforced by a significant causal relationship of CRPcognition, observed in the relationship between CRP risk level and cognitive function. In the present study, CRP level in the high-risk condition compared to that in low risk was related to lower score in Stroop interference and slower memory retrieval latency in DMS suggesting that high CRP risk might causes deleterious effect on cognitive function especially such as inhibitory control and working memory. Evidence suggests that the Stroop interference is widely used as frontal lobe functioning (Stuss, Floden, Alexander, Levine, \& Katz, 2001), however, higher CRP level is associated with cerebral microstructural disintegration that predominantly affects frontal pathways and corresponding worse executive function (Wersching et al., 2010). In addition, impaired memory assessed with Selective Reminding Test including delayed recall and delayed recognition was related to higher CRP level. The higher CRP level as a systemic inflammatory marker leads to impaired endothelial function, which was associated with vascular dementia (Vicenzini et al., 2007). Systemic inflammatory markers are also independently associated with impaired cerebral blood flow (Novak et 
al., 2006 ) and cerebrovascular reserve (Davenport, Hogan, Eskes, Longman, \& Poulin, 2012), particularly in the hippocampus, which is important to memory (Semmler, Okulla, Sastre, Dumitrescu-Ozimek, \& Heneka, 2005). Combined, these studies, and our adjusted regression analysis, demonstrated that CRP might be associated with impairing cognitive function and could be use as a biomarker of cognitive dysfunction in individuals without dementia.

\subsection{Health Indices and Cognitive Function}

Physical activity and cardiorespiratory fitness examined were significantly related to executive function and working memory. In the present study, although our adjusted regression analysis didn't reveal a significant relationship between BMI and any variable of cognitive function tests possibly due to our small sample size relatively, there might be a trend showing that CRP might be a potential mediator of the relationship between BMI and working memory as the strong negative association that BMI has with CRP appears to contribute to cognitive dysfunction. Otherwise, other studies confirmed that obesity was associated with lower cognitive functioning measured as tests of learning and memory and more cognitive deficit if there are more cardiovascular risk factors involved (Elias, 2003) and obesity itself has a compounding negative impact on the brain by increasing low-grade systemic inflammation (Smith, Hay, Campbell, \& Trollor, 2011). The impact of obesity and vascular risk on cognition needs to be further investigated, given obesity has risen dramatically in adults (Ogden et al., 2014). Physical activity level had a significant and positive direct effect on cognitive function, particularly including Stroop interference and working memory, independent of CRP. This finding is aligned with other findings demonstrating the beneficial effects of physical activity on brain function during adulthood, particularly frontal lobe-mediated cognitive processes including planning, inhibition, and working memory (Drummond et al., 2005; Nieder \& Miller, 2004) as well as the protecting effect of physical activity on cognitive decline in nondemented participants followed for 1-12 years (Sofi et al., 2011). Further, these data from adjusted regression analysis suggests that the most powerful effects of health indices on working memory through CRP occur from the level of cardiorespiratory fitness. The cardiorespiratory fitness level had both direct effect and indirect effect through CRP on working memory. This result suggests that those having higher cardiorespiratory fitness level could have a greater working memory directly and indirectly by reducing CRP level. A recent study also confirmed that cardiorespiratory fitness was positively related to neuropsychological performance, particularly, including memory (Wendell et al., 2014).

In the current study, the data from adjusted regression analysis revealed the significant relationship of any health indices and CRP with sustained attention was not observed, however, cardiorespiratory fitness and physical activity was significantly correlated with sustained attention (Hillman et al., 2005). Possible explanation is that rather than comparing the baseline reaction time in PVT, this test was appropriate for the pre-test and posttest (Dinges et al., 1997; Dinges \& Powell, 1985) as mostly used in acute exercise-induced the sustained attention improvement (Mahon et al., 2013; 
Pontifex, Saliba, Raine, Picchietti, \& Hillman, 2012)

The beneficial effects of physical exercise-induced improvements in cardiorespiratory fitness on cognitive functions have been supported by several potential mechanisms including enhancement of endothelial function and decline of arterial stiffness, oxidative stress, and vascular inflammation, which increase resting cerebral blood flow and improve function of cerebrovascular reserve (Davenport et al., 2012) and incline of brain volume and size, particularly in hippocampus (Erickson et al., 2011), and release of neurotrophins, particularly, brain-derived neurotrophic factor (BDNF), which promote neuronal growth and survival and mediate learning and memory (Vaynman, Ying, \& Gomez-Pinilla., 2004). A recent study also confirmed that cardiorespiratory fitness and BDNF was positively associated with recognition memory as suggested by an interaction between physical exercise and BNDF that predicts recognition memory accuracy (Whiteman et al., 2014).

These findings suggest that the complex interaction among health indices, CRP, and cognitive function are crucial to our understanding for implementing future strategies to tackle inadequate physical activity/cardiorespiratory fitness levels and poorer body weight control on cognitive health in young adults.

\subsection{Delimitations and Limitations}

This study has several strengths and potential limitations. The strengths of our study include the most accurate measure of cardiorespiratory fitness through metabolic cart on cycle ergometer to measure VO2max. This study benefitted from accurate information on VO2max, which we were, therefore, able to examine the valid and reliable association of cardiorespiratory fitness with CRP and cognitive function. Although the variable of cognitive tasks was restricted to the variation in demographic variable (i.e. age, gender, race), our study was used for the variable measures controlling for the demographic characteristics to reduce potential confounding effects. Lastly, as the study used neuropsychological test battery including crystallized intelligence, executive function, attention, and working memory, we were, therefore, able to identify the relationship of major cognitive domain with health indices and vascular risk in young adults.

The limitations of our study include data collected on physical activity based on a self-reported data, which undermines the accurate classification of physical activity. The self-reported measure of physical activity was derived from a 7day physical activity recall, but the physical activity instruments have been acceptable measurement characteristics. Future studies should utilize more objective measures of physical activity such as accelerometer and activity monitor although this would be difficult from financial perspective for population studies.

\subsection{Implications}

The present findings represent a novel investigation of how health indices influence physical fitness and mediate cognitive performance. Continued study is valuable as it has substantial public health implications. Sedentary lifestyles increase vascular risk and deplete cognitive function. These findings confirm the beneficial effects of physical exercise-induced improvements in cardiorespiratory fitness on vascular 
risk and cognitive functions particularly in working memory. The second decade of life represents the cognitive peak; unlike previous studies there was a differentiated cognitive performance among the participants, based on health the presence of health risk such as elevated CRP. This physical activity has both physical and cognitive benefits among young adults. As such, increasing physical activity participation in moderate to vigorous physical activity to improve cardiorespiratory fitness and to promote healthy BMI may reduce or suppress vascular risk with potential positive effects on cognitive health. 


\section{REFERENCES}

ACSM. (2013). ACSM's guidelines for exercise testing and prescription (9th ed). Lippincott Williams and Wilkins, New York.

Albert, M. A., Glynn, R. J., \& Ridker, P. M. (2004). Effect of physical activity on serum C-reactive protein. Am J Cardiol., 15(93(2)), 221-225.

Author, Centeio, E., Author, J., Barcelona, J. M., Glowacki, E. M., Calvert, H. G., \& Nicksic, H. M. (2014). VII. The history of physical activity and academic performance research: informing the future. Monogr Soc Res Child Dev., 79(4), 119-148.

Author, Brothers, R. M., Author, Nicksic, N. H., Glowacki, E., Harrison, M., \& Dongen, D. V. (2013). Health Indices and Cognitive Performance in Emerging Adults. J Nov Physiother, 4(189).

Chudasama, \& Yogita. (2010). Delayed (Non) Match-to-Sample Task. In I. Stolerman (Ed.), Encyclopedia of Psychopharmacology (pp. 372-372): Springer Berlin Heidelberg.

Church, T., Barlow, C., Earnest, C., Kampert, J., Priest, E., \& Blair, S. (2002). Associations Between Cardiorespiratory Fitness and CReactive Protein in Men. Arterioscler Thromb Vasc Biol, 22(22), 1869-1876.

Craig, C. L., Marshall, A. L., Sjostrom, M., Bauman, A. E., Booth, M. L., Ainsworth, B. E., Oja, P. (2003). International physical activity questionnaire: 12-country reliability and validity. Med Sci Sports Exerc, 35(8), 1381-1395. doi: 10.1249/01.MSS.0000078924.61453.F B
Davenport, M. H., Hogan, D. B., Eskes, G. A., Longman, R. S., \& Poulin, M. J. (2012). Cerebrovascular Reserve: The Link Between Fitness and Cognitive Function? Exercise and Sport Sciences Reviews, 40(3), 153-158. doi: 10.1097/JES.0b013e3182553430

Dinges, D., Pack, F., Williams, K., Gillen, K., Powell, J., Ott, G., . . . Pack, A. (1997). Cumulative sleepiness, mood disturbance, and psychomotor vigilance performance decrements during a week of sleep restricted to 4-5 hours per night. Sleep, 20(4), 267-277.

Dinges, D., \& Powell, J. (1985). Microcomputer analyses of performance on portable, simple visual RT task during sustained operations. . Behav Res Methods Instrum, 17, 652-655.

Drummond, S., Bischoff-Grethe, A., Dinges, D., Ayalon, L., Mednick, S., \& Meloy, M. (2005). The neural basis of the psychomotor vigilance task. Sleep, 28(9), 1059-1068.

Elias, M. F. E., P. K. Sullivan, L. M. Wolf, P. A. D'Agostino, R. B. (2003). Lower cognitive function in the presence of obesity and hypertension: the Framingham heart study. Int $J$ Obes Relat Metab Disord, 27(2), 260-268.

Erickson, K. I., Voss, M. W., Prakash, R. S., Basak, C., Szabo, A., Chaddock, L., . . Kramer, A. F. (2011). Exercise training increases size of hippocampus and improves memory. Proceedings of the National Academy of Sciences, 108(7), 3017-3022. doi: 10.1073/pnas. 1015950108

Ford, E. S. (2002). Does exercise reduce inflammation? Physical activity and Creactive protein among U.S. adults. Epidemiology, 13, 561-568. 
Ge, X., Xu, X., Feng, C., Wang, Y., Li, Y., \& Feng, B. (2013). Relationships among serum C-reactive protein, receptor for advanced glycation products, metabolic dysfunction, and cognitive impairments. BMC Neurosci, 13(110).

Golden, C. (1978). Stroop Color and Word Test. . A Manual for Clinical and Experimental 414 Uses. Skoelting, Chicago, Illinois, 1-32.

Hayes, S., Forman, D., \& Verfaellie, M. (2014). Cardiorespiratory Fitness Is Associated With Cognitive Performance in Older But Not Younger Adults. $J$ Gerontol B Psychol Sci Soc Sci., gbul67.

Heinrich, P. C., Castell, J. V., \& Andus, T. (1990). Interleukin-6 and the acute phase response. Biochem J Biochem J, $265,621-636$.

Hillman, C. H., Author, D. M., \& Buck, S. M. (2005). Aerobic Fitness and Neurocognitive Function in Healthy Preadolescent Children. Medicine \& Science in Sports \& Exercise, 37(11), 1967-1974. doi:

10.1249/01.mss.0000176680.79702.ce

Hotamisligil, G. S., Arner, P., Caro, J. F., Atkinson, R. L., \& Spiegelman, B. M. (1995). Increased adipose tissue expression of tumor necrosis factoralpha in human obesity and insulin resistance. J Clin Invest, 95, 2409-2415.

Jae, S., Heffernan, K., Yoon, E., Le, M., Fernhall, B., \& Park, W. (2009). The Inverse Association between Cardiorespiratory Fitness and CReactive Protein Is Mediated by Autonomic Function: A Possible Role of the Cholinergic Antiinflammatory Pathway. . Molecular Medicine, 15(910), 291-296.
Jialal, I., Devaraj, S., \& Venugopal, S. K. (2004). C-Reactive Protein: Risk Marker or Mediator in Atherothrombosis? Hypertension, 44, 611.

Kasapis, C., \& Thompson, P. D. (2005). The Effects of Physical Activity on Serum C-Reactive Protein and Inflammatory Markers. J Am Coll Cardiol., 45(10), 1563-1569.

Kaufman, A. S., and Kaufman, N. L. (1990). Kaufman Brief Intelligence Test. Circle Pines, $M N$.

Komulainen, P., Lakka, T., Kivipelto, M., Hassinen, M., Penttilä, L., Helkala, E., . .

Rauramaa, R. (2007). Serum high sensitivity $\mathrm{C}$-reactive protein and cognitive function in elderly women. Age Ageing, 36(4), 443-448. doi: 10.1093/ageing/afm051

LaMonte, M., Durstine, J., Yanowitz, F., Lim, T., DuBose, K., Davis, P., \& Ainsworth, B. (2002). Cardiorespiratory fitness and C-reactive protein among a tri-ethnic sample of women. Circulation, 106(4), 403-406.

Lohman, T. G., Roche, A. F., \& Martorell, R. (1988). Anthropometric standardization reference manual. Champaign, IL : Human Kinetics Books.

Mahon, A. D., Dean, R. S., McIntosh, D. E., Marjerrison, A. D., Cole, A. S., Woodruff, M. E., \& Lee, M. P. (2013). Acute Exercise Effects on Measures of Attention and Impulsivity in Children with Attention Deficit/Hyperactivity Disorder. Journal of Educational and Developmental Psychology, 3(2).

Mueller, S. T., \& Piper, B. J. (2014). The Psychology Experiment Building Language (PEBL) and PEBL Test 
Battery. J Neurosci Methods, 222, 250259. doi:

10.1016/j.jneumeth.2013.10.024

Nieder, A., \& Miller, E. K. (2004). A parieto-frontal network for visual numerical information in the monkey. PNAS, 101, 7457-7462.

Noble, J., Manly, J., Schupf, N., Tang, M., Mayeux, R., \& Luchsinger, J. (2010). Association of C-Reactive Protein With Cognitive Impairment. . Arch Neurol, 67(1), 87-92.

Novak, V., Last, D., Alsop, D. C., Abduljalil, A. M., Hu, K., Lepicovsky, L., ... Lipsitz, L. A. (2006 ). Cerebral blood flow velocity and periventricular white matter hyperintensities in type 2 diabetes. Diabetes Care, 29(7), 1529-1534.

Ogden, Carroll, M. D., Kit, B. K., \& Flegal, K. M. (2014). Prevalence of childhood and adult obesity in the United States, 2011-2012. Journal of the American Medical Association, 311(8), 806-814.

Pate, R., Pratt, M., Blair, S., Haskell, W., Macera, C., Bouchard, C., . . . King, A. (1995). Physical activity and public health. A recommendation from the Centers for Disease Control and Prevention and the American College of Sports Medicine. JAMA, 273(5), 402-407.

Pontifex, M. B., Saliba, B. J., Raine, L. B., Picchietti, D. L., \& Hillman, C. H. (2012). Exercise Improves Behavioral, Neurocognitive, and Scholastic

Performance in Children with Attention-Deficit/Hyperactivity Disorder. J Pediatr, 16(3), 543-551.

Rosenberg, D., Bull, F., Marshall, A., Sallis, J., \& Bauman, A. (2008).

Assessment of sedentary behavior with the International Physical Activity Questionnaire. J Phys Act Health, 5(1), S30-44.

Sanchez-Cubillo, I., Perianez, J. A., Adrover-Roig, D., Rodriguez-Sanchez, J. M., Rios-Lago, M., Tirapu, J., \& Barcelo, F. (2009). Construct validity of the Trail Making Test: role of taskswitching, working memory, inhibition/interference control, and visuomotor abilities. J Int Neuropsychol Soc, 15(3), 438-450. doi: $10.1017 / \mathrm{S} 1355617709090626$

Semmler, A., Okulla, T., Sastre, M., Dumitrescu-Ozimek, L., \& Heneka, M. T. (2005). Systemic inflammation induces apoptosis with variable vulnerability of different brain regions. J Chem Neuroanat, 30(2-3), 144-157.

Smith, Servesco, A., \& Edwards, J. (2008). Exploring the validity of the comprehensive trail making test. Clin. Neuropsychol., 22, 507-518.

Smith, E., Hay, P., Campbell, L., \& Trollor, J. N. (2011). A review of the association between obesity and cognitive function across the lifespan: implications for novel approaches to prevention and treatment. Obes Rev, 12(9), 740-755. doi: 10.1111/j.1467789X.2011.00920.X

Sofi, F., Valecchi, D., Bacci, D., Abbate, R., Gensini, G. F., Casini, A., \& Macchi, C. (2011). Physical activity and risk of cognitive decline: a metaanalysis of prospective studies. $J$ Intern Med, 269(1), 107-117. doi: 10.1111/j.1365-2796.2010.02281.x

Stroop, J. (1935). Studies of interference in serial verbal reactions. J. Exp. Psychol., 18, 643-662.

Stuss, D. T., Floden, D., Alexander, M. 
P., Levine, B., \& Katz, D. (2001). Stroop performance in focal lesion patients: Dissociation of processes and frontal lobe lesion location. Neuropsychologia, 39(771-786).

USDHHS. (2008a). 2008 physical activity guidelines for Americans. Washington, DC: DHHS. 2008., http://www.health.gov/paguidelines/guid elines/default.aspx.

USDHHS. (2008b). Healthy People 2010 [online] http://www.

healthypeople.gov/Document (2000).

Vaynman, S., Ying, Z., \& GomezPinilla., F. (2004). Hippocampal BDNF mediates the efficacy of exercise on synaptic plasticity and cognition. Eur $J$ Neurosci, 20(10), 2580-2590.

Vicenzini, E., Ricciardi, M. C., Altieri, M., Puccinelli, F., Bonaffini, N., Di Piero, V., \& Lenzi, G. L. (2007). Cerebrovascular reactivity in degenerative and vascular dementia: a transcranial Doppler study. Eur Neurol., 58(2), 84-89.

Wendell, C. R., Gunstad, J., Waldstein, S. R., Wright, J. G., Ferrucci, L., \& Zonderman, A. B. (2014).

Cardiorespiratory Fitness and Accelerated Cognitive Decline With Aging. The Journals of Gerontology Series A: Biological Sciences and Medical Sciences, 69(4), 455-462. doi: 10.1093/gerona/glt144

Wersching, H., Duning, T., Lohmann, H., Mohammadi, S., Stehling, C., Fobker, M., ...

Knecht, S. (2010). Serum C-reactive protein is linked to cerebral microstructural integrity and cognitive function (Vol. 74): Neurology.
Whiteman, A. S., Young, D. E., He, X., Chen, T. C., Wagenaar, R. C., Stern, C. E., \& Schona, K. (2014). Interaction between serum BDNF and aerobic fitness predicts recognition memory in healthy young adults. Behavioural Brain Research, 259(302-312).

Zhu, D., Schreiner, P., Yaffe, K., Bryan, N., Launer, L., Sternfeld, B. (2014). Cardiorespiratory fitness and cognitive function in middle age: the CARDIA study. Neurology, 15(82), 1339-1346.

Zhu, Y. S., Tarumi, T., Tseng, T. Y., Palmer, D. M., Levine, B. D., \& Zhang, R. (2013). Cerebral vasomotor reactivity during hypo- and hypercapnia in sedentary elderly and Masters athletes. J Cereb Blood Flow Metab, 33(8), 1190-1196. doi: $10.1038 / \mathrm{jcbfm} .2013 .66$ 